placement of the annuloplasty ring may be controversial. We prefer to preserve the architecture of the intact anulus without placing the ring at the bridging tissue. To the best of our knowledge, a case with valve repair in isolated DOMV with two equal orifices has not been reported previously. Though the follow-up is still short term in our case, we conclude that valve repair is the choice of treatment for mitral regurgitation in isolated DOMV.

REFERENCES

1. Bano-Rodrigo A, Van Praagh S, Trowitzsch E, Van Praagh R. Double-orifice mitral valve: a study of 27 cases with develop mental, diagnostic and surgical considerations. Am J Cardiol 1988;61:152-60.
2. Trowitzsch E, Bano-Rodrigo A, Burger B, Colan SD, Sanders SP. Two-dimensional echocardiographic findings in double orifice mitral valve. J Am Coll Cardiol 1985;6:383-7.

3. Cascos AS, Rabago P, Sokolowski M. Case report: duplication of the tricuspid valve. Br Heart J 1967;29:943-5.

4. Kenaan G, Neufeld HN, Deutsch V, Shem-Tov A. Isolated congenital double-orifice mitral valve. Isr J Med Sci 1974;10: 743-6.

5. Kawahira M, Sanada J, Kataoka H, Nakamura K, Hashimoto S. A case of double orifice mitral valve complicated by ruptured chordae tendineae. Proc Jap Soc Ulc Med 1992;41: 311-2.

6. Amano J, Suzuki A. Surgical treatment of duplication of the mitral valve. J Cardiovasc Surg 1986;27:323-7.

\title{
PERCUTANEOUS TRANSVENOUS ANGIOPLASTY OF A STENOSED BICAVAL ANASTOMOSIS AFTER ORTHOTOPIC CARDIAC TRANSPLANTATION
}

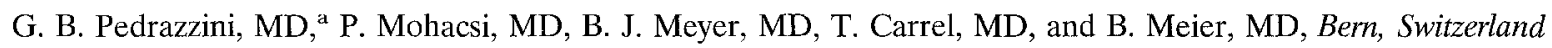

Orthotopic cardiac transplantation is the treatment of choice for patients with end-stage heart failure. The operative technique used at most transplantation centers was developed in the early 1960 s by Lower and Shumway ${ }^{1}$ with a canine model. This original technique includes two atrial anastomoses and anastomosis of the aorta and pulmonary artery. Main advantages of this classical procedure are its simplicity, short operative time, and low prevalence of perioperative and postoperative technical complications.

Mild to moderate regurgitation of the atrioventricular valves (mainly of the tricuspid valve and to a lesser degree of the mitral valve), however, is a common postoperative clinical and echocardiographic finding. These morphologic and functional changes of the normal donor heart are thought to occur because of a postoperative modification in the atrial geometry. Moreover, the technique of biatrial anastomoses seems to increase the occurrence of sinus node dysfunction. ${ }^{2}$

To prevent these potential complications, an alternative technique with bicaval anastomoses rather than the right atrial suture was recently reintroduced by several groups

From the Department of Cardiology ${ }^{\mathrm{a}}$ and Clinic for Thoracic and Cardiovascular Surgery, ${ }^{\mathrm{b}}$ University Hospital, Bern, Switzerland.

Paul Mohacsi supported by a grant from the Swiss National Research Foundation (grant No. 31-39695.1).

Received for publication Feb. 13, 1996; accepted for publication March 25, 1996.

J Thorac Cardiovasc Surg 1996;112:1667-9

Copyright 1996 by Mosby-Year Book, Inc.

$0022-5223 / 96 \$ 5.00+0 \quad 12 / 54 / 73791$ performing for transplantation. This technical refinement was originally described by Webb and colleagues ${ }^{3}$ in 1959 . The anatomic geometry and the sinus node function seem to be better preserved, but this technique can lead to significant stenosis of the venous anastomoses even when an interrupted suture technique is used. This report discusses the case of a patient with a severe stenosis of the superior vena cava (SVC) 2 weeks after heart transplantation. The condition was treated successfully with percutaneous balloon angioplasty.

Cardiac transplantation was performed in a 52-year-old white man because of end-stage dilated cardiomyopathy. Instead of biatrial anastomoses, separate SVC and inferior vena caval anastomoses were performed with interrupted 4.0 polypropylene sutures. A caliber mismatch between the donor and recipient SVCs was noted, with the recipient's SVC three times as large as the donor's SVC. After operation, immediate sinus rhythm was present and no significant atrioventricular regurgitation was demonstrated.

The postoperative course was uneventful, with the exception of cellular rejection (ISHLT $3 \mathrm{~A}$ ) on postoperative day 14 , which was successfully treated with corticosteroids. An endomyocardial biopsy sample was difficult to obtain, however, because it was nearly impossible to introduce a guide wire through the SVC into the right atrium. After several attempts, successful introduction of a $6 \mathrm{~F}$ catheter revealed a pressure gradient of $10 \mathrm{~mm} \mathrm{Hg}$ across the stenosis (Fig. 1). The tight stenosis was confirmed by SVC angiography, and it was decided to use percutaneous angioplasty to dilate the anastomosis at the SVC level.

For this purpose, a $9 \mathrm{~F}$ sheath and a Trefoil $3 \times 6 \mathrm{~mm}$ balloon (Schneider AG, Bulach, Switzerland) was used. 

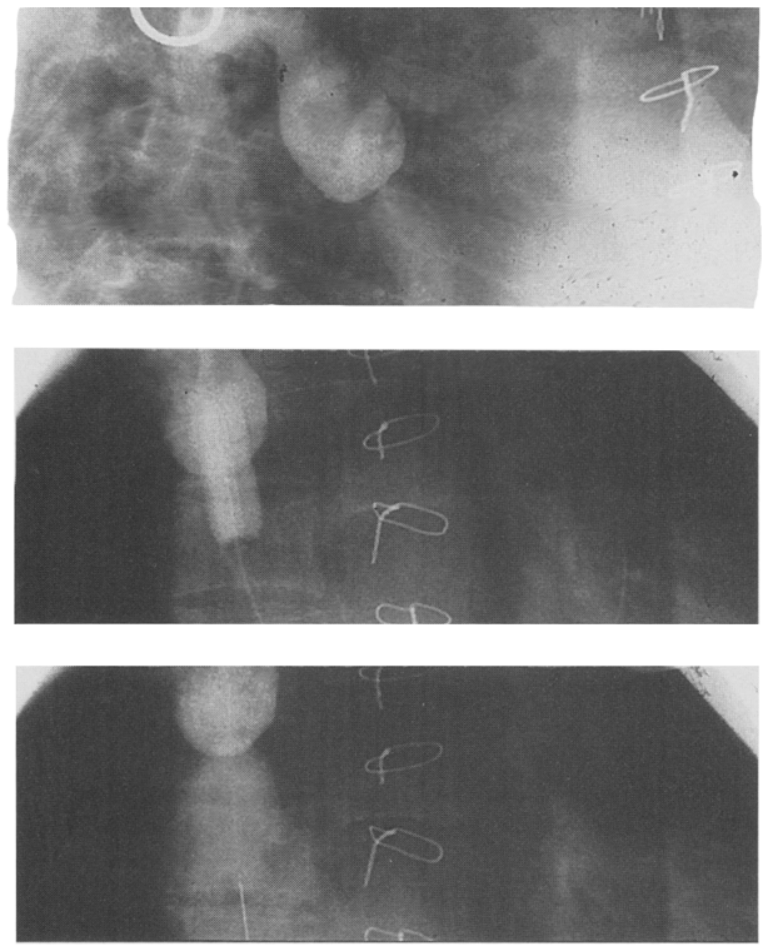

Fig. 1. Radiograph of 52-year-old man 2 weeks after heart transplantation with bicaval anastomoses for dilated cardiomyopathy. Top, Severe stenosis of the SVC anastomosis; middle, Trefoil $3 \times 6 \mathrm{~mm}$ balloon inflated in the stenosis; bottom, condition after successful dilatation.

The balloon was inflated at $11 \mathrm{~atm}$. On angiography after dilatation, the stenosis was seen to be improved (Fig. 1); a residual pressure gradient of $4 \mathrm{~mm} \mathrm{Hg}$, which persisted during follow-up studies, was noted. Angiographic assessment at 1 week and 3 months did not reveal any restenosis (Fig. 2). Clinically, the patient is doing well and has no signs of venous congestion of the upper body.

The modified orthotopic transplantation technique with bicaval anastomoses represents a valid alternative to classic orthotopic heart transplantation. This procedure maintains the atrial geometry and therefore may provide better atrioventricular valvular function with less regurgitation. ${ }^{2,4-6}$ Despite some potential morphologic and functional advantages, the technique of bicaval anastomosis may lead to a stenosis of the vena cava at the level of the suture. This problem may be emphasized by a difference in caliber between the donor and recipient veins and also by the purse-string effect caused by use of a continuous suture technique.

The interrupted suture technique (running suture of the posterior wall and interrupted stiches of the anterior wall) may partially reduce the prevalence of significant stenosis of the vena caval anastomosis. Because of the larger caliber of the inferior vena cava, this complication has not been described at that location.

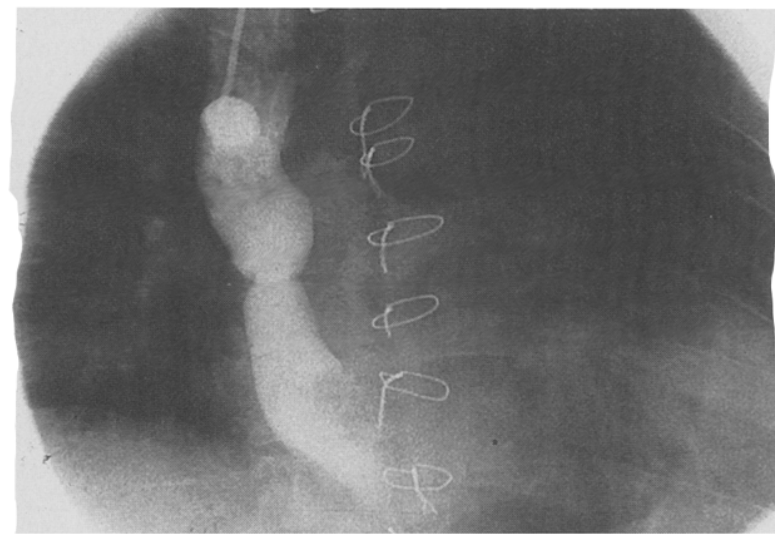

Fig. 2. Follow-up radiograph 3 months after treatment.

Although our patient did not have clinical signs of upper venous congestion, the bicaval anastomosis was stenosed enough to cause a significant pressure gradient and difficulties in obtaining endomyocardial biopsy samples. Because of the favorable appearance of the stenosis, it was decided to perform percutaneous transvenous angioplasty even though the risk of a balloon intervention seemed higher early after heart transplantation.

This procedure avoided a second operation and allowed us to obtain an immediate decrease in the pressure gradient and an easier passage through the SVC to the right heart cavities. Furthermore, the excellent follow-up results after balloon angioplasty in this case demonstrate the feasibility and safety of this procedure, even early after transplantation. Angiographic assessment and pressure gradient measurements at 1 week and at 3 months revealed no recurrent stenosis, and endomyocardial biopsies are actually easy to perform. An alternative to angioplasty would have been the repair of the anastomosis with a temporary shunt between the cranial part of the SVC and the right atrium.

Orthotopic cardiac transplantation with bicaval anastomoses represents an attractive alternative to the conventional technique. Even when interrupted suture lines are used, however, the bicaval anastomotic technique may occasionally lead to stenoses of the vena cava, which may necessitate operative treatment. For these patients, percutaneous balloon angioplasty represents an attractive treatment alternative. In the case of a significant caliber mismatch between the donor and recipient SVCs, a classic biatrial anastomosis should be considered.

\section{REFERENCES}

1. Lower RR, Shumway NE. Studies on orthotopic homotransplantation of the canine heart. Surg Forum 1962;11:18-9.

2. Sievers HH, Leik R, Jahnke A, Pertry A, Kraatz EG, Herrmann $G$, et al. Bicaval versus atrial anastomoses in cardiac transplantation. J Thorac Cardiovasc Surg 1994;108:780-4. 
3. Webb WR, Howard HS, Neely WA. Practical method of homologous cardiac transplantation. J Thorac Surg 1959;37: 361-6.

4. Dreyfus G, Jebora V, Mikaileanu S, Carpentier A. Total orthotopic heart transplantation: an alternative technique to the standard technique. Ann Thorac Surg 1991;52:1181-4.
5. Laske A, Carrel T, Niederhäuser U, Pasic M, Segesser LK, Jenni R, et al. Modified operation technique for orthotopic heart transplantation. Eur J Cardiothorac Surg 1995;9:120-6.

6. Leih R, Jahnke A, Kraatz E, Sievers H. Cardiovascular dynamic and dimension after bicaval and standard cardiac transplantation. Ann Thorac Surg 1995;59:1495-500.

\title{
COMPLETE INTRAOPERATIVE DISLODGMENT OF A ST. JUDE MEDICAL MECHANICAL HEART VALVE HEMODYNAMIC PLUS* VALVE SEWING CUFF
}

\author{
Nicola Vitale, MD, ${ }^{a}$ Donato Serena, MD, ${ }^{\text {a }}$ Corrado Fondacone, MD, ${ }^{\mathrm{a}}$ Alfonso Agnino, MD, ${ }^{\mathrm{a}}$ Pasquale Cicala, MD, \\ and Luigi de Luca T.S., MD, Bari, Italy
}

Mechanical disruption of bileaflet heart valves has previously been described. ${ }^{1-4}$ The St. Jude Medical Mechanical Heart Valve Hemodynamic Plus (HP) valve (St. Jude Medical, Inc., St. Paul, Minn.) is a prosthesis designed to reduce transvalvular gradients by increasing valvular area. In 1995, a randomized Italian multicenter study on the hemodynamic performance in the aortic position of the St. Jude Medical HP valve versus the St. Jude Medical standard valve (St. Jude Medical, Inc.) was started. We describe a case of complete intraoperative dislodgment of a St. Jude Medical HP sewing cuff that occurred during the course of that study. We believe this event to be the first reported case of sewing cuff slip-off of a St. Jude Medical valve in the literature.

A 61-year-old man with coronary artery disease and severe aortic regurgitation was referred to our department for aortic valve replacement and coronary artery bypass grafting. After admission to our unit, he gave informed consent for his enrollment in the multicenter study on the hemodynamic performance in the aortic position of the St. Jude Medical HP valve versus the St. Jude Medical standard valve.

At operation, once standard cardiopulmonary bypass had been instituted with two-stage venous cannululation, the aorta was crossclamped and opened. Surgical inspection revealed severe aortic regurgitation with advanced thinning of the valve cusps and no calcification. After valvular excision, the aortic anulus was measured with both St. Jude Medical standard and HP sizers; a $23 \mathrm{~mm}$ diameter was found to be adequate and a $23 \mathrm{~mm}$ St. Jude Medical HP valve was randomly selected.

From the Departments of Cardiac Surgery and Anesthesiology, Medical School, University of Bari, Bari, Italy.

Received for publication April 2, 1996; accepted for publication April 9, 1996.

J Thorac Cardiovasc Surg 1996;112:1669-71

*St. Jude Medical Mechanical Heart Valve Hemodynamic Plus is a trade name of St. Jude Medical, Inc., St. Paul, Minn.

Copyright $(\mathcal{C} 1996$ by Mosby-Year Book, Inc.

$0022-5223 / 96 \$ 5.00+0 \quad \mathbf{1 2 / 5 4 / 7 4 0 5 1}$
The valve implantation was performed in our usual manner. Interrupted U-shaped 2-0 Tevdek sutures (Deknatel Division, Fall River, Mass.) with no pledgets were passed at supraannular level and then through the sewing cuff. The prosthesis was oriented with the axis perpendicular to the ventricular septum. During these stages, the prosthesis was always encased in its holder and held by the assistant surgeon, so no stress was applied to the sewing cuff while the stitches were being placed through the cuff, nor was any rotational force exerted on the valve.

When the prosthesis was inserted while it was being slid down to its supraannular position, the entire sewing cuff was dislodged, causing the sewing cuff to slip completely off of the pyrolitic carbon ring (Figs. 1 and 2). The insertion maneuver was carried out by the first surgeon, who always held the prosthesis in its holder so that no stress was applied to the cuff.

After this breakdown, stitches were quickly removed from the sewing cuff and then mounted on free needles to replace the St. Jude Medical HP valve with a CarboMedics valve (CarboMedics Inc., Austin, Texas) of the same diameter. At the end of valve implantation, coronary artery bypass grafting with the left internal thoracic artery on the left anterior descending coronary artery was also performed.

The postoperative course was uneventful. At the time of this writing, the patient is well and free of complications. The broken St. Jude Medical HP valve has been returned to the manufacturer for examination.

Structural dysfunction of bileaflet valve prosthesis generally concerns leaflet rupture with subsequent embolization. ${ }^{1-4}$ To the best of our knowledge, this is the first reported case of intraoperative dislodgment of the sewing cuff.

The problem occurred during implantation of a 23 aortic St. Jude Medical HP valve in a patient with severe aortic regurgitation. The sewing cuff on the St. Jude Medical HP series of valves is a small horizontal flange designed to be sutured above the anulus. Only the carbon orifice ring is placed into the anulus. The sewing cuff is held in place by six suture windings running tightly around the inner ring. To increase the orifice area, the sewing cuff was thinned by eliminating all cuff material from the 